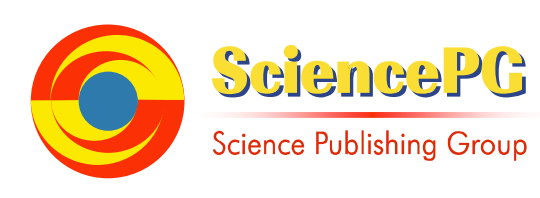

\title{
A cubic Bézier model with shape parameters
}

\author{
Juncheng Li \\ Department of Mathematics, Hunan University of Humanities, Science and Technology, Loudi, China
}

Email address:

lijuncheng82@126.com

To cite this article:

Juncheng Li. A Cubic Bézier Model with Shape Parameters. Applied and Computational Mathematics. Vol. 3, No. 6, 2014 , pp. 343-348. doi: $10.11648 /$ j.acm.20140306.19

\begin{abstract}
A novel extension of the cubic Bézier curve with two shape parameters is presented in this work. The proposed curve is still a cubic polynomial model, which has simpler structure than other similar models. The proposed curve has the same properties with the usual cubic Bézier curve and its shape can be adjusted by altering values of the two shape parameters while the control points are fixed. With the two shape parameters, the proposed curve can approach to its control polygon farther or closer. The corresponding surface with four shape parameters has the similar properties with the proposed curve and enjoys the shape adjustable property.
\end{abstract}

Keywords: Cubic Bézier Curve, Cubic Polynomial, Shape Parameter, Shape Adjustment

\section{Introduction}

In Computer Aided Geometric Design (CAGD), the cubic Bézier curve and surface have been widely used for geometric modeling. However, when the control points are given, the shapes of the usual cubic Bézier curve and surface cannot be changed. With the development of geometric design industry, shapes of curves and surface often need to be changed freely. Hence, shapes of the usual cubic Bézier curve and surface cannot be modified limit their practical applications in geometric modeling. For relieving the default of the usual cubic Bézier curve and surface, the Bézier-like curves and surfaces with shape parameters have been paid more and more attention by many researchers.

At present, in order to introduce shape parameters to the usual cubic Bézier curve and surface, the most commonly used methods have two kinds. One is to construct the quasi cubic Bézier curves and surfaces with shape parameters based on the space with trigonometric or hyperbolic functions, such as [1-8], another is to construct the high-degree Bézier curves and surfaces with shape parameters by increasing the degree of the Bernstein basis, such as [9-14]. Although those methods can effectively realize the shape adjustment of the curves and surfaces by altering the values of the shape parameters, the structure complexity is thereupon increased. The Bézier curve and surface with multiple shape parameters of the same degree [15] was a novel method, but the curve and surface did not have the strict symmetry that the usual Bézier curve and surface have. The main purpose of this work is to present another novel extension of the cubic Bézier curve and surface with shape parameters. The shape of the proposed curve and surface can be adjusted by altering values of the shape parameters when the control points are fixed. More important, the proposed curve and surface are still cubic polynomial models and has the same properties with the usual cubic Bézier models.

The rest of this paper is organized as follows. In Section 2, the cubic Bézier basis with two shape parameters $(\alpha \beta$-Bézier basis for short) is constructed, and some properties of the basis are given. In Section 3, the corresponding curve with two shape parameters $(\alpha \beta$-Bézier curve for short) is defined, the properties of the $\alpha \beta$-Bézier curve are discussed and effects of the shape parameters on $\alpha \beta$-Bézier curve are studied. In Section 4, the corresponding surface with four shape parameters ( $\alpha \beta$-Bézier surface for short) is presented. A short conclusion is given in Section 5.

\section{2. $\alpha \beta$-Bézier Basis}

The construction process of a class of cubic Bézier basis with two shape parameters $\alpha$ and $\beta$ ( $\alpha \beta$-Bézier basis for short) are given as below.

Suppose the $\alpha \beta$-Bézier basis will be constructed is expressed as follows, 


$$
\left[\begin{array}{llll}
f_{0}(t) & f_{1}(t) & f_{2}(t) & f_{3}(t)
\end{array}\right]=\left[\begin{array}{llll}
1 & t & t^{2} & t^{3}
\end{array}\right] M
$$

where $t \in[0, \alpha](0<\alpha \leq 1)$, and $M$ is an undetermined $4 \times 4$ matrix.

Derivation calculus to Eq. (1), then

$$
\left[\begin{array}{llll}
f_{0}^{\prime}(t) & f_{1}^{\prime}(t) & f_{2}^{\prime}(t) & f_{3}^{\prime}(t)
\end{array}\right]=\left[\begin{array}{llll}
0 & 1 & 2 t & 3 t^{2}
\end{array}\right] M
$$

The $\alpha \beta$-Bézier basis is hoped to satisfy the similar properties with the usual cubic Bernstein basis functions at the end point. Let $t=0$ and $t=\alpha$ in Eq. (1) and Eq. (2) respectively, then

$$
\begin{aligned}
& {\left[\begin{array}{llll}
1 & 0 & 0 & 0
\end{array}\right]=\left[\begin{array}{llll}
1 & 0 & 0 & 0
\end{array}\right] M} \\
& {\left[\begin{array}{llll}
0 & 0 & 0 & 1
\end{array}\right]=\left[\begin{array}{llll}
1 & \alpha & \alpha^{2} & \alpha^{3}
\end{array}\right] M} \\
& {\left[\begin{array}{llll}
-\beta & \beta & 0 & 0
\end{array}\right]=\left[\begin{array}{llll}
0 & 1 & 0 & 0
\end{array}\right] M} \\
& {\left[\begin{array}{lllll}
0 & 0 & -\beta & \beta
\end{array}\right]=\left[\begin{array}{llll}
0 & 1 & 2 \alpha & 3 \alpha^{2}
\end{array}\right] M}
\end{aligned}
$$

From Eq. (3) to Eq. (6), then

$$
\left[\begin{array}{cccc}
1 & 0 & 0 & 0 \\
0 & 0 & 0 & 1 \\
-\beta & \beta & 0 & 0 \\
0 & 0 & -\beta & \beta
\end{array}\right]=\left[\begin{array}{cccc}
1 & 0 & 0 & 0 \\
1 & \alpha & \alpha^{2} & \alpha^{3} \\
0 & 1 & 0 & 0 \\
0 & 1 & 2 \alpha & 3 \alpha^{2}
\end{array}\right] M
$$

Solving Eq. (7) and taking the result to Eq. (1), the $\alpha \beta$-Bézier basis can be defined as follows.

Definition 1. For two arbitrary selected real values of $\alpha(0<\alpha \leq 1)$ and $\beta(0<\beta \leq 3)$, the following four functions of $t(0 \leq t \leq \alpha)$ are called the cubic Bézier basis with two shape parameters $\alpha$ and $\beta$ ( $\alpha \beta$-Bézier basis for short),

$$
\left\{\begin{array}{l}
f_{0}(t)=\frac{1}{\alpha^{3}}(\alpha-t)^{2}[(\alpha-t)+(3-\alpha \beta) t] \\
f_{1}(t)=\frac{\beta}{\alpha^{2}} t(\alpha-t)^{2} \\
f_{2}(t)=\frac{\beta}{\alpha^{2}} t^{2}(\alpha-t) \\
f_{3}(t)=\frac{1}{\alpha^{3}} t^{2}[(\alpha-t)(3-\alpha \beta)+t]
\end{array}\right.
$$

Theorem 1. The $\alpha \beta$-Bézier basis defined as Eq. (8) has the following properties,

(a) Nonnegativity: $f_{i}(t) \geq 0 \quad(i=0,1,2,3)$.

(b) Degeneration: For $\alpha=1$ and $\beta=3$, the $\alpha \beta$-Bézier basis degenerates into the usual cubic Bernstein basis.

(c) Monotonicity: For fix $t \in[0, \alpha]$ and $\alpha \in(0,1], f_{0}(t)$ and $f_{3}(t)$ are monotonically decreasing about $\beta, f_{1}(t)$ and $f_{2}(t)$ are monotonically increasing about $\beta$.

(d) Partition of unity:

$$
f_{0}(t)+f_{1}(t)+f_{2}(t)+f_{3}(t) \equiv 1 .
$$

(e) Symmetry:

$$
f_{i}(t ; \alpha, \beta)=f_{3-i}(\alpha-t ; \alpha, \beta) \quad(i=0,1,2,3) .
$$

(f) Properties at the endpoints:

$$
\begin{gathered}
\left\{\begin{array}{l}
f_{0}(0)=1, \quad f_{1}(0)=f_{2}(0)=f_{3}(0)=0 \\
f_{0}(\alpha)=f_{1}(\alpha)=f_{2}(\alpha)=0, \quad f_{3}(\alpha)=1
\end{array}\right. \\
\left\{\begin{array}{l}
f_{0}^{\prime}(0)=-\beta, \quad f_{1}^{\prime}(0)=\beta, \\
f_{2}^{\prime}(0)=0, \quad f_{3}^{\prime}(0)=0, \\
f_{0}^{\prime}(\alpha)=0, \quad f_{1}^{\prime}(\alpha)=0, \\
f_{2}^{\prime}(\alpha)=-\beta, \quad f_{3}^{\prime}(\alpha)=\beta .
\end{array}\right.
\end{gathered}
$$

Proof. (a) For $0 \leq t \leq \alpha, 0<\alpha \leq 1$ and $0<\beta \leq 3$, then $\alpha-t \geq 0,3-\alpha \beta \geq 0$. From Eq. (8), $f_{i}(t) \geq 0 \quad(i=0,1,2,3)$ follow obviously.

(b) For $\alpha=1$ and $\beta=3$, the $\alpha \beta$-Bézier basis can be expressed as follows,

$$
\left\{\begin{array}{l}
f_{0}(t)=(1-t)^{3} \\
f_{1}(t)=3(1-t)^{2} t \\
f_{2}(t)=3(1-t) t^{2} \\
f_{3}(t)=t^{3}
\end{array}\right.
$$

Eq. (11) is the expression of the usual cubic Bernstein basis exactly, which shows that the $\alpha \beta$-Bézier basis degenerates into the cubic Bernstein basis functions when $\alpha=1$ and $\beta=3$.

(c) For $t \in[0, \alpha]$ and $\alpha \in(0,1]$, then

$$
\begin{gathered}
\frac{\mathrm{d} f_{0}}{\mathrm{~d} \beta}=-\frac{t}{\alpha^{2}}(\alpha-t)^{2} \leq 0, \frac{\mathrm{d} f_{1}}{\mathrm{~d} \beta}=\frac{t}{\alpha^{2}}(\alpha-t)^{2} \geq 0, \\
\frac{\mathrm{d} f_{2}}{\mathrm{~d} \beta}=\frac{t^{2}}{\alpha^{2}}(\alpha-t) \geq 0, \frac{\mathrm{d} f_{3}}{\mathrm{~d} \alpha}=-\frac{t^{2}}{\alpha^{2}}(\alpha-t) \leq 0 .
\end{gathered}
$$

Hence, $f_{0}(t)$ and $f_{3}(t)$ are monotonically decreasing about $\beta, f_{1}(t)$ and $f_{2}(t)$ are monotonically increasing about $\beta$.

By simple deduction, the remaining cases follow obviously.

Fig. 1 and Fig. 2 show curves of the $\alpha \beta$-Bézier basis for different values of $\alpha$ and $\beta$. In Fig. 1 , the value of $\beta$ is set for $\beta=3$, and the value of $\alpha$ is set for $\alpha=0.4$ (solid lines), $\alpha=0.8$ (dotted lines) and $\alpha=1.0$ (dashed lines) respectively. In Fig. 2, the value of $\alpha$ is set for $\alpha=1.0$, and the value of $\beta$ is set for $\beta=1.0$ (solid lines), $\beta=2.0$ (dotted lines) and $\beta=3.0$ (dashed lines) respectively. 


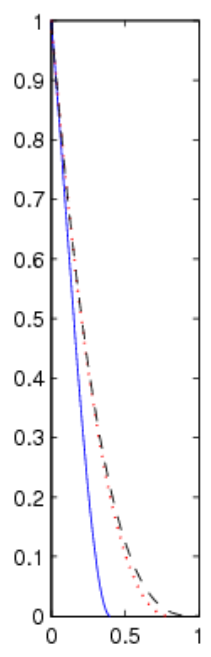

$f_{0}(t)$
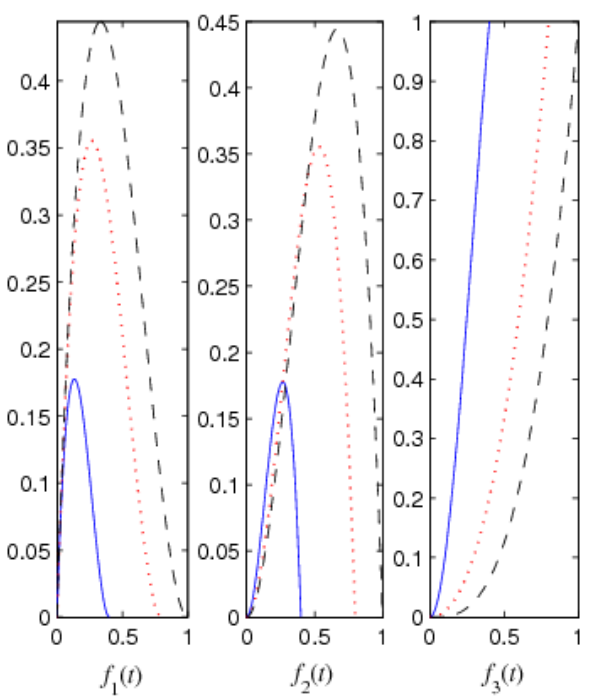

Figure 1. Curves of the $\alpha \beta$-Bézier basis for different values of $\alpha$

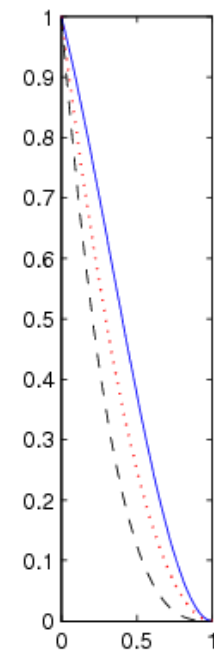

$f_{0}(t)$

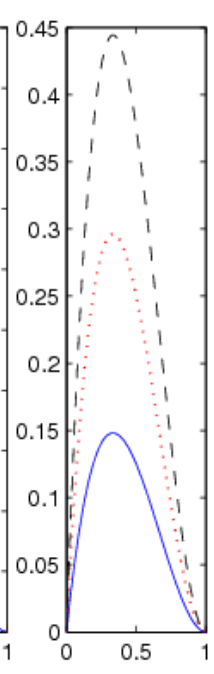

$f_{1}(t)$

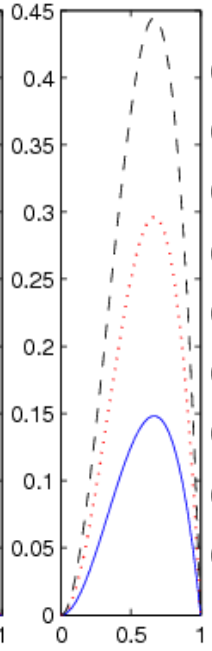

$f_{2}(t)$

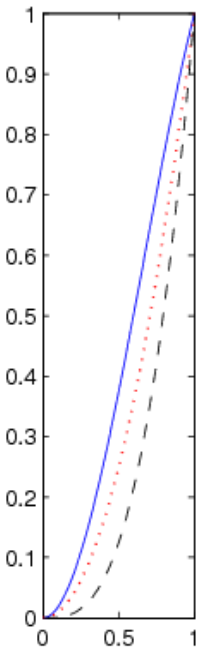

$f_{3}(t)$
Figure 2. Curves of the $\alpha \beta$-Bézier basis for different values of $\beta$

\section{3. $\boldsymbol{\alpha} \boldsymbol{\beta}$-Bézier Curve}

\subsection{Definition and Properties of the $\alpha \beta$-Bézier Curve}

On the base of the $\alpha \beta$-Bézier basis, the corresponding curve with two shape parameters can be defined as follows.

Definition 2. Given $\boldsymbol{p}_{i}(i=0,1,2,3)$ are four control points in $R^{2}$ or $R^{3}$, for $0 \leq t \leq \alpha, 0<\alpha \leq 1$ and $0<\beta \leq 3$, then

$$
\boldsymbol{B}(t)=\sum_{i=0}^{3} f_{i}(t) \boldsymbol{p}_{i}
$$

is called the cubic Bézier curve with two shape parameters $\alpha$ and $\beta$ ( $\alpha \beta$-Bézier curve for short), where $f_{i}(t)(i=0,1,2,3)$ are the $\alpha \beta$-Bézier basis defined as Eq. (8).

Remark 1. The curve defined as Eq. (12) can be reparametrized to a new curve by $\boldsymbol{R}(u)=\boldsymbol{B}(\alpha u)$. Then $\boldsymbol{R}(u)$ is defined on a fixed interval $[0,1]$. For given control points $\boldsymbol{p}_{i}(i=0,1,2,3)$, both $\boldsymbol{B}(t)$ and $\boldsymbol{B}(u)$ represent the same curve. The curve with $\boldsymbol{B}(t)$ is used in the following discussion.

From the properties of the $\alpha \beta$-Bézier basis and definition of the $\alpha \beta$-Bézier curve, some properties of the $\alpha \beta$-Bézier curve can be obtained as follows.

Theorem 2. The $\alpha \beta$-Bézier curve defined as Eq. (12) has the following properties,

(a) Terminal properties: The curve interpolates the first and the end control points and tangent to the first and the end edges of the control polygon, i.e.,

$$
\left\{\begin{array}{l}
\boldsymbol{B}(0)=\boldsymbol{p}_{0} \\
\boldsymbol{B}(\alpha)=\boldsymbol{p}_{3} \\
\boldsymbol{B}^{\prime}(0)=\beta\left(\boldsymbol{p}_{1}-\boldsymbol{p}_{0}\right) \\
\boldsymbol{B}^{\prime}(\alpha)=\beta\left(\boldsymbol{p}_{3}-\boldsymbol{p}_{2}\right)
\end{array}\right.
$$

(b) Symmetry: For the same shape parameter $\alpha \quad(0<\alpha \leq 1)$ and $\beta \quad(0<\beta \leq 3)$, both $\boldsymbol{p}_{i}$ and $\boldsymbol{p}_{3-i}(i=0,1,2,3)$ define the same curve in a different parameterization, i.e.,

$$
\boldsymbol{B}\left(t ; \boldsymbol{p}_{0}, \boldsymbol{p}_{1}, \boldsymbol{p}_{2}, \boldsymbol{p}_{3}\right)=\boldsymbol{B}\left(\alpha-t ; \boldsymbol{p}_{3}, \boldsymbol{p}_{2}, \boldsymbol{p}_{1}, \boldsymbol{p}_{0}\right)
$$

where $0 \leq t \leq \alpha$.

(c) Geometric invariant and affine invariance: Location and shape of the curve depend only on the four control points $\boldsymbol{p}_{i}(i=0,1,2,3)$, the parameter $\alpha$ and $\beta$, regardless of the choice of coordinate system, i.e., shape of the curve will keep unchanged after rotation and coordinate translation. In addition, after implementing affine transformation to the control points, the new curve will correspond to the same affine transformation curve.

(d) Convex hull property: A curve with $\boldsymbol{B}(t)$ must lie inside its control polygons span by $\boldsymbol{p}_{i}(i=0,1,2,3)$.

Proof. (a) From Eq. (9) and Eq. (12), then

$$
\begin{gathered}
\boldsymbol{B}(0)=f_{0}(0) \boldsymbol{p}_{0}+f_{1}(0) \boldsymbol{p}_{1}+f_{2}(0) \boldsymbol{p}_{2}+f_{3}(0) \boldsymbol{p}_{3}=\boldsymbol{p}_{0} \\
\boldsymbol{B}(\alpha)=f_{0}(\alpha) \boldsymbol{p}_{0}+f_{1}(\alpha) \boldsymbol{p}_{1}+f_{2}(\alpha) \boldsymbol{p}_{2}+f_{3}(\alpha) \boldsymbol{p}_{3}=\boldsymbol{p}_{3}
\end{gathered}
$$

From Eq. (10) and Eq. (12), then

$$
\begin{gathered}
\boldsymbol{B}^{\prime}(0)=f_{0}^{\prime}(0) \boldsymbol{p}_{0}+f_{1}^{\prime}(0) \boldsymbol{p}_{1}+f_{2}^{\prime}(0) \boldsymbol{p}_{2}+f_{3}^{\prime}(0) \boldsymbol{p}_{3} \\
\quad=-\beta \boldsymbol{p}_{0}+\beta \boldsymbol{p}_{1}=\beta\left(\boldsymbol{p}_{1}-\boldsymbol{p}_{0}\right) \\
\boldsymbol{B}^{\prime}(\alpha)=f_{0}^{\prime}(\alpha) \boldsymbol{p}_{0}+f_{1}^{\prime}(\alpha) \boldsymbol{p}_{1}+f_{2}^{\prime}(\alpha) \boldsymbol{p}_{2}+f_{3}^{\prime}(\alpha) \boldsymbol{p}_{3} \\
\quad=-\beta \boldsymbol{p}_{3}+\beta \boldsymbol{p}_{2}=\beta\left(\boldsymbol{p}_{3}-\boldsymbol{p}_{2}\right)
\end{gathered}
$$

(b) For the same shape parameter $\alpha \quad(0<\alpha \leq 1)$ and $\beta(0<\beta \leq 3)$, from symmetry of the $\alpha \beta$-Bézier basis, then 


$$
\begin{aligned}
& \boldsymbol{B}\left(\alpha-t ; \boldsymbol{p}_{3}, \boldsymbol{p}_{2}, \boldsymbol{p}_{1}, \boldsymbol{p}_{0}\right) \\
& =f_{0}(\alpha-t) \boldsymbol{p}_{3}+f_{1}(\alpha-t) \boldsymbol{p}_{2}+f_{2}(\alpha-t) \boldsymbol{p}_{1}+f_{3}(\alpha-t) \boldsymbol{p}_{0} \\
& =f_{3}(t) \boldsymbol{p}_{3}+f_{2}(t) \boldsymbol{p}_{2}+f_{1}(t) \boldsymbol{p}_{1}+f_{0}(t) \boldsymbol{p}_{0} \\
& =\boldsymbol{B}\left(t ; \boldsymbol{p}_{0}, \boldsymbol{p}_{1}, \boldsymbol{p}_{2}, \boldsymbol{p}_{3}\right)
\end{aligned}
$$

(c) Because of the parametric form of the curve, this property follows obviously.

(d) This property follows since the $\alpha \beta$-Bézier basis satisfies nonnegative property and sums to one which are shown in Theorem 1.

Remark 2. Theorem 2 shows that the $\alpha \beta$-Bézier curve inherits the same properties with the usual cubic Bézier curve. Particularity, the $\alpha \beta$-Bézier curve degenerates into the usual cubic Bézier curve when $\alpha=1$ and $\beta=3$. Therefore, the $\alpha \beta$-Bézier curve is an extension of the usual cubic Bézier curve.

Remark 3. There are many cubic Bézier-like curves with shape parameters had been constructed by some researchers. They introduce the shape parameters through modifying the polynomial functions of the usual cubic Bézier curve to trigonometric or hyperbolic functions [1-8], or increasing the degree of the usual cubic Bézier curve [9-14]. The shapes of those curves can be adjusted by altering values of the shape parameters, but the structure complexity is thereupon increased. Although Xiang, et al [15] constructed a cubic Bézier curve with shape parameters of the same degree, the curve did not have the strict symmetry that the usual cubic Bézier curve has. In contrast with those similar curves, the $\alpha \beta$-Bézier curve presented in this work has the following characteristic,

(a) In contrast with the quasi cubic Bézier curve with the parameter [1-8], the $\alpha \beta$-Bézier curve is a cubic polynomial curve. Hence, structure of the $\alpha \beta$-Bézier curve is simpler than those curves based on the space with trigonometric or hyperbolic functions.

(b) In contrast with the high-degree Bézier curve with the parameter [9-14], the $\alpha \beta$-Bézier curve is still a cubic polynomial model. Hence, formula complexity of the $\alpha \beta$-Bézier curve is simpler than those curves constructed by increasing the degree of the Bernstein basis functions.

(c) In contract with the Bézier curve with multiple parameters of the same degree [15], the $\alpha \beta$-Bézier curve satisfies strict symmetry that the usual cubic Bézier curve has. Hence, the $\alpha \beta$-Bézier curve is more suitable in practical engineering than the curve didn't satisfy strict symmetry.

\subsection{Effects of the Shape Parameters on $\alpha \beta$-Bézier Curve}

When the four control points are fixed, shape of the usual cubic Bézier curve cannot be changed, while shape of the proposed curve can be adjusted by altering values of the shape parameter $\alpha(0<\alpha \leq 1)$ and $\beta(0<\beta \leq 3)$. Effects of the shape parameters on the $\alpha \beta$-Bézier curve approaches to its polygon are shown as follows.

Theorem 3. Suppose $\boldsymbol{p}_{i} \quad(i=0,1,2,3)$ are not collinear, and $\boldsymbol{p}_{0}, \boldsymbol{p}_{3}$ lie on the same side of edge $\boldsymbol{p}_{0} \boldsymbol{p}_{3}$, the shape parameters $\alpha$ and $\beta$ have the following effects on the $\alpha \beta$-Bézier curve,

(a) When $\beta$ is fixed, the $\alpha \beta$-Bézier curve approaches closer to its control polygon as $\alpha$ increases.

(b) When $\alpha$ is fixed, the $\alpha \beta$-Bézier curve approaches closer to its control polygon as $\beta$ increases

(c) The $\alpha \beta$-Bézier curve approaches closer to its control polygon as $\alpha$ and $\beta$ increase simultaneously.

Proof. Let $\boldsymbol{p}^{*}=\frac{\boldsymbol{p}_{1}+\boldsymbol{p}_{2}}{2}$, from Eq. (12), then

$$
\boldsymbol{B}\left(\frac{\alpha}{2}\right)-\boldsymbol{p}^{*}=\frac{4-\alpha \beta}{8}\left(\boldsymbol{p}_{0}-\boldsymbol{p}_{1}-\boldsymbol{p}_{2}+\boldsymbol{p}_{3}\right)
$$

where $0<\alpha \leq 1,0<\beta \leq 3$.

Taking the norm in Eq. (13), then

$$
\left\|\boldsymbol{B}\left(\frac{\alpha}{2}\right)-\boldsymbol{p}^{*}\right\|=\frac{4-\alpha \beta}{8}\left\|\boldsymbol{p}_{0}-\boldsymbol{p}_{1}-\boldsymbol{p}_{2}+\boldsymbol{p}_{3}\right\|
$$

From Eq. (14), then

(a) When $\beta$ is fixed, since $\frac{4-\alpha \beta}{8}$ decreases as $\alpha$ increases, the $\alpha \beta$-Bézier curve approaches closer to its control polygon with the increase of $\alpha$.

(b) When $\alpha$ is fixed, since $\frac{4-\alpha \beta}{8}$ decreases as $\beta$ increases, the $\alpha \beta$-Bézier curve approaches closer to its control polygon with the increase of $\beta$.

(c) Since $\frac{4-\alpha \beta}{8}$ decreases as $\alpha$ and $\beta$ simultaneously increase, the $\alpha \beta$-Bézier curve approaches closer to its control polygon with the simultaneous increase of $\alpha$ and $\beta$.

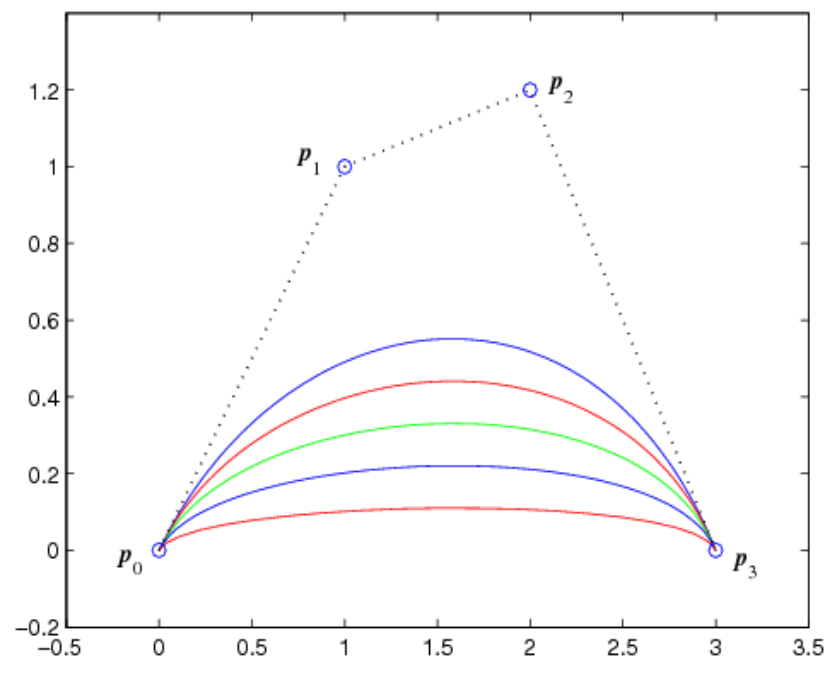

Figure 3. Effects of $\alpha$ on the $\alpha \beta$-Bézier curve

Fig. 3 shows the $\alpha \beta$-Bézier curve corresponding to the same control polygon by fixing $\beta=2$ and setting $\alpha=0.2,0.4,0.6,0.8,1.0$ respectively from outside to inside.

Fig. 4 shows the $\alpha \beta$-Bézier curve corresponding to the same control polygon by fixing $\alpha=0.5$ and setting 
$\beta=0.5,1.0,1.5,2.0,2.5,3.0$ respectively from outside to inside.

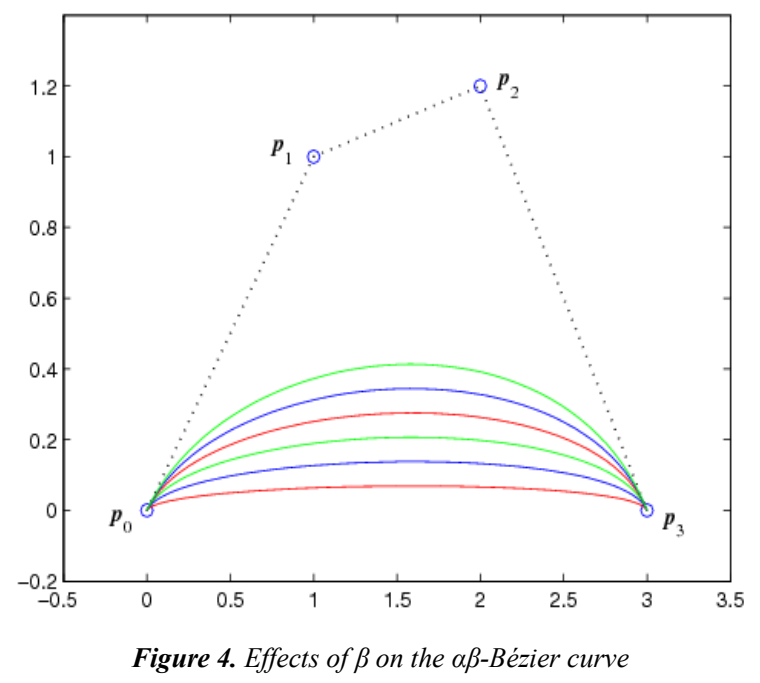

Fig. 5 shows the $\alpha \beta$-Bézier curve corresponding to the same control polygon by setting $\alpha=0.2, \beta=1.0, \alpha=0.4, \beta=1.5$, $\alpha=0.6, \beta=2.0 \quad, \quad \alpha=0.8, \beta=2.5 \quad, \quad \alpha=1.0, \beta=3.0$ respectively from outside to inside.

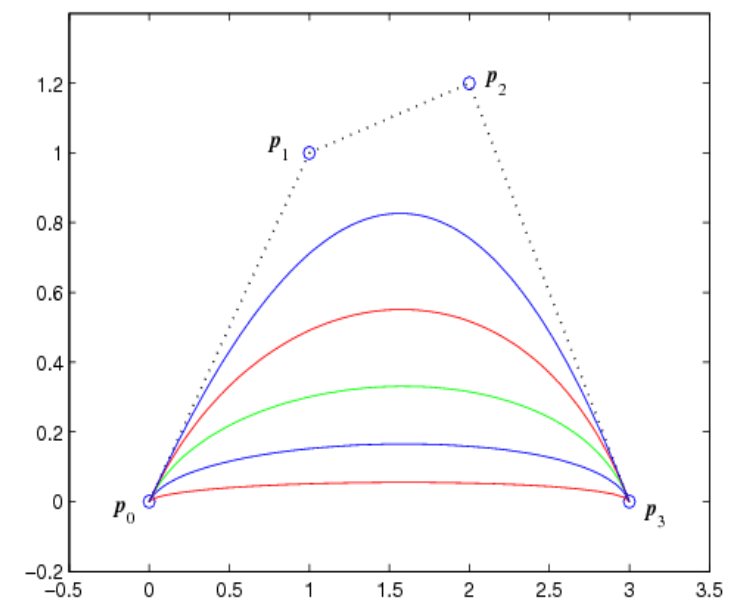

Figure 5. Effects of $\alpha$ and $\beta$ on the $\alpha \beta$-Bézier curve

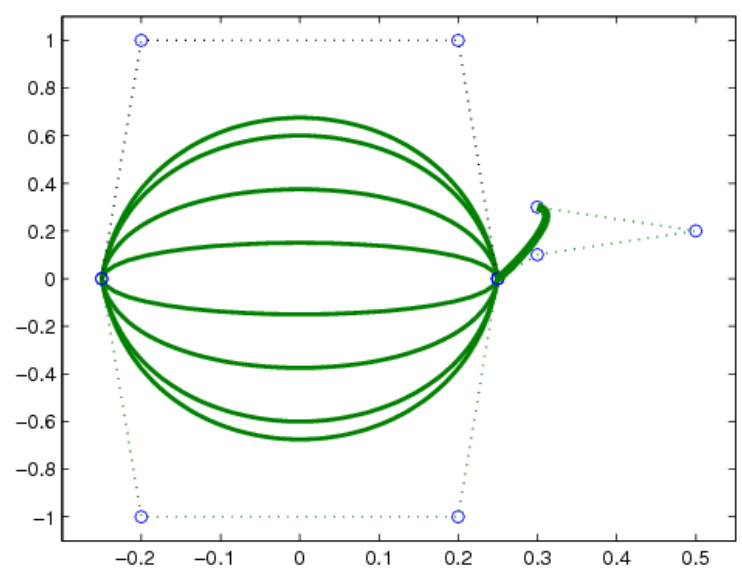

Figure 6. Watermelon-shaped pattern generated by the $\alpha \beta$-Bézier curve
The watermelon-shaped pattern generated by altering values of the shape parameters $\alpha$ and $\beta$ is shown in Fig. 6 .

\section{4. $\alpha \beta$-Bézier Surface}

Using tensor product, the corresponding surface with four shape parameters can be defined as follows.

Definition 3. Given $\boldsymbol{p}_{i, j}(i, j=0,1,2,3)$ are $4 \times 4$ control points in $R^{3}, f_{i}(u)\left(0 \leq u \leq \alpha_{1}, 0<\alpha_{1} \leq 1,0<\beta_{1} \leq 3\right)$ and $f_{j}(v)\left(0 \leq u \leq \alpha_{2}, 0<\alpha_{2} \leq 1,0<\beta_{2} \leq 3\right)$ are the basis defined according to Eq. (8), then

$$
\boldsymbol{B}(u, v)=\sum_{i=0}^{3} \sum_{j=0}^{3} f_{i}(u) f_{j}(v) \boldsymbol{p}_{i, j}
$$

is called the cubic Bézier surface with four shape parameters $\alpha_{i}$ and $\beta_{i} \quad(i=1,2) \quad(\alpha \beta$-Bézier surface for short $)$.

The $\alpha \beta$-Bézier surface defined as Eq. (15) has symmetry, geometric invariant and convex hull property that the usual cubic Bézier surface has. Particularity, the $\alpha \beta$-Bézier surface degenerates into the usual Bézier surface when $\alpha_{i}=1$ and $\beta_{i}=3(i=1,2)$. When the control mesh is fixed, shape of the $\alpha \beta$-Bézier surface can be adjusted by altering values of the four shape parameters $\alpha_{i}$ and $\beta_{i} \quad(i=1,2)$.

Fig. 7 shows the $\alpha \beta$-Bézier surface corresponding to the same control mesh by setting different values of the shape parameters $\alpha_{i}=\alpha$ and $\beta_{i}=\beta \quad(i=1,2)$, where (a) $\alpha=0.5, \beta=1.0$, (b) $\alpha=1.0, \beta=1.0$, (c) $\alpha=0.8, \beta=2.0$, (d) $\alpha=0.8, \beta=3.0$.

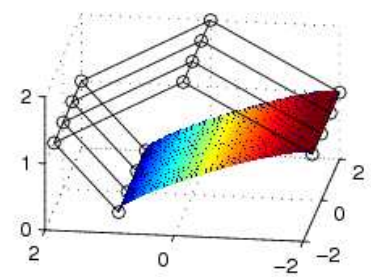

(a)

(c)

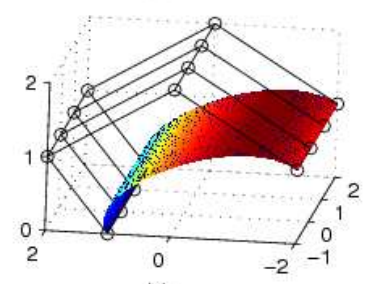

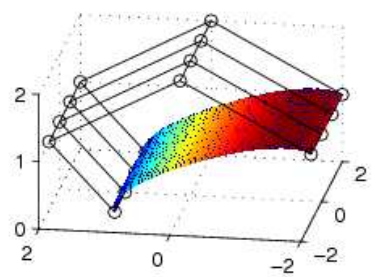

(b)

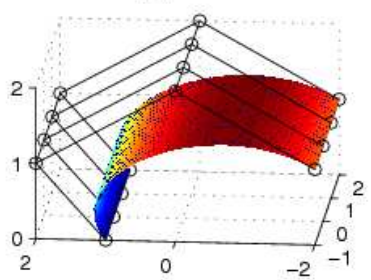

(d)
Figure 7. The $\alpha \beta$-Bézier surface for different values of shape parameters

\section{Conclusions}

As mentioned above, the $\alpha \beta$-Bézier curve not only has the same properties with the usual cubic Bézier curve, but also can be easily adjusted by altering values of the shape parameters when the control points are fixed. More important, the $\alpha \beta$-Bézier curve is still a cubic polynomial model, which has 
simpler structure than other similar models. The $\alpha \beta$-Bézier surface also has the same properties with the usual cubic Bézier surface and its shape can be adjusted by altering values of the shape parameters while the control mesh is kept unchanged. Because there is nearly no difference in structure between the $\alpha \beta$-Bézier models and the usual cubic Bézier models, it is no difficult to adopt the $\alpha \beta$-Bézier models to a $\mathrm{CAD} / \mathrm{CAM}$ system that already uses the usual cubic Bézier models.

For practical applications of $\alpha \beta$-Bézier models in geometric modeling, it is clear that some special algorithms need to be established. Some interesting results in this area will be discussed in the following study.

\section{Acknowledgments}

This work was supported by the Hunan Provincial Natural Science Foundation of China under grant number 13JJ6081 and Scientific Research Fund of Hunan Provincial Education Department of China under grant number 14B099. The author is also very grateful to the Hunan provincial key construction discipline "Computer Application Technology" of Hunan University of Humanities, Science and Technology of China.

\section{References}

[1] J. W. Zhang, "C-Bézier curves and surfaces", Graphical Models and Image Processing, vol. 61, no. 1, pp. 2-15, 1999.

[2] Q. Y. Chen and G. Z. Wang, "A class of Bézier-like curves", Computer Aided Geometric Design, vol. 20, no. 1, pp. 29-39, 2003.

[3] J. W. Zhang, F. L. Krause and H. Y. Zhang. "Unifying C-curves and $\mathrm{H}$-curves by extending the calculation to complex numbers", Computer Aided Geometric Design, vol. 22, no. 9, pp. 865-883, 2005.

[4] X.-A. Han, Y. C. Ma and X. L. Huang, "The cubic trigonometric Bézier curve with two shape parameters",
Applied Mathematical Letters, vol. 22, no. 2, pp. 226-231, 2009.

[5] J. C. Li, D. B. Zhao, B. J. Li and G. H. Chen, "A family of quasi-cubic trigonometric curves", Journal of Information and Computational Science, vol. 7, no. 13, pp. 2847-2854, 2010.

[6] X. L. Han and Y. P. Zhu, "Curve construction based on five trigonometric blending functions", BIT Numerical Mathematics, vol. 52, no. 4, pp. 953-979, 2012.

[7] J. C. Li, "A class of cubic trigonometric Bézier curve with a shape parameter", Journal of Information and Computational Science, vol. 10, no.10, pp. 3071-3078, 2013.

[8] U. Bashir, M. Abbsa and J. M.Ali, "The $\mathrm{G}^{2}$ and $\mathrm{C}^{2}$ rational quadratic trigonometric Bézier curve with two shape parameters with applications", Applied Mathematics and Computation, vol. 219. no. 20, pp. 10183-10197, 2013.

[9] W. T. Wang and G. Z. Wang, "Bézier curves with shape parameters", Journal of Zhejiang University SCIENCE A, vol. 6, no. 6, pp. 497-501, 2005.

[10] X.-A. Han, Y. C. Ma and X. L.Huang, "A novel generalization of Bézier curve and surface", Journal of Computational and Applied Mathematics, vol. 217, no. 1, pp. 180-193, 2008.

[11] L. Q. Yang and X M. Zeng, "Bézier curves and surfaces with shape parameters", International Journal of Computer Mathematics, vol. 86, no. 7, pp. 1253-1263, 2009.

[12] L. L. Yan and Q. F. Liang, "An extension of the Bézier model", Applied Mathematics and Computation, vol. 218, no. 6, pp. 2863-2879, 2011.

[13] J. Chen and G. J. Wang, "A new type of the generalized Bézier curves", Applied Mathematics: A Journal of Chinese Universities, vol. 26, no. 1, pp. 47-56, 2011.

[14] Y. P. Zhu and X. L. Han, "A class of $\alpha \beta \gamma$-Bernstein-Bézier basis functions over triangular domain", Applied Mathematics and Computation, vol. 220, no. 17, pp. 446-454, 2013.

[15] T. N. Xiang, Z. Liu, W. F. Wang and P. Jiang, "A novel extension of Bézier curves and surfaces of the same degree", Journal of Information and Computational Science, vol. 7, no. 10, pp. 2080-2089, 2010. 